\title{
CUBIC NONLINEAR FRACTIONAL SCHRÖDINGER EQUATION WITH CONFORMABLE DERIVATIVE AND ITS NEW TRAVELLING WAVE SOLUTIONS
}

\author{
Hami Gündoğdu, Ömer Faruk Gözü̈kızıl \\ Sakarya University, Faculty of Arts and Sciences, Department of Mathematics \\ Sakarya, Turkey \\ hamigundogdu@sakarya.edu.tr,farukg@sakarya.edu.tr
}

Received: 27 January 2021; Accepted: 3 June 2021

\begin{abstract}
In the present paper, the fractional-order cubic nonlinear Schrödinger equation is considered. The Schrödinger equation with time and space fractional derivative is studied at the same time. Different types of travelling wave solutions including the kink solution, soliton solution, periodic solution, and singular solution for the mentioned equation are obtained by using the Jacobi elliptic functions expansion method. It is shown that the solutions turn into the exact solutions when the fractional orders go to 1 . This method can be relied on gaining the solutions to time or space fractional order partial differential equations as well as ordinary ones. Throughout this work, the fractional derivative is given in a conformable sense.
\end{abstract}

MSC 2010: 35R11, 35C07, 35C08

Keywords: cubic nonlinear fractional Schrödinger equation, travelling wave solutions, kink solution, soliton solution

\section{Introduction}

Linear and nonlinear partial differential equations, N/LPDEs, have been utilized to model physical events occurring in science and engineering. In addition to N/LPDEs, fractional ones have been used to convert real-life problems into mathematical language. So, the fractional-order partial differential equations, FPDEs, holds a significant place in a lot of areas of modern science such as chemistry, physics, biology, electricity, electronic, viscoelasticity, plasma physics, dynamical system, genetics algorithms, signal processing, robotic technology, telecommunication, economics and finance [1-3].

Scientists have placed their focus on acquiring the solutions of FPDEs since they take places in the areas mentioned above. Motivated by this fact, scientists have been looking for establishing methods to get the solutions of FPDEs. The tanh-coth method $[4,5]$, the exp-function method [6], the (G'/G) expansion method $[7,8]$, the Laplace-Adomian decomposition method (LADM) [9, 10], and Jacobi elliptic 
function expansion method [11-13] can be given as examples for the most well-known methods.

One of the most significant PDEs is the cubic nonlinear Schrödinger equation, introduced by Laskin [14], as follows:

$$
i u_{t}+u_{x x}+\mu|u|^{2} u=0,
$$

where $u(x, t)$ is a complex function of $x$ and $t$. The equation (1) models the propagation of pulses in fibers excluding fiber loss. It also symbolizes the evolution of envelope for modulated nonlinear wave groups. Moreover, it is seen in nonlinear wave propagation in dispersive and inhomogeneous media. Furthermore, it plays important roles in several areas of physics including water waves, plasma physics, fiber optics, quantum mechanics, and so on. For further information, see [14].

Over the last few decades, there has been a lot of research on how to gain the exact solutions for the Schrödinger equations. Biswas [15] implemented the semiinverse variational method to the nonlinear Schrödinger's equation for getting soliton solutions. For the soliton solutions of the nonlinear Schrödinger equation with time-dependent coefficients, the simplest equation approach is used by Eslami et al. [16]. Mirzazadeh et al. [17] looked for the topological solitons of the nonlinear Schrödinger equation with dual-power law nonlinearity by using the $\left(G^{\prime} / G\right)$ expansion method. Exact solutions of the nonlinear Schrödinger equation were found by Inc and Ates [18] with the help of the extended Jacobi elliptic function expansion method.

Some researchers employed other methods to seek soliton solutions of the nonlinear Schrödinger's equation with different forms of nonlinearities [19-21].

Now, we present the space-time fractional Schrödinger equation as follows

$$
i D_{t}^{\gamma} u+D_{x}^{2 \beta} u+\mu|u|^{2} u=0, \quad 0<\gamma, \beta \leq 1, \quad t>0,
$$

where $\mu \neq 0$ is a real parameter. The equation is called to be attractive if $\mu>0$. On the contrary, it is called to be repulsive when $\mu<0$. For detailed information, see [22-24].

In the Eq. (2), the fractional derivative is in the conformable sense. The reason why the conformable derivative was used is that it has some important features given in section 2. Sharing same propeties with the integer order derivative gives the opportunity to understand the complex beahviour of real evolution process. The conformable derivate is commonly used in fractional order mathematical models due to these properties.

The main purpose of this paper is to obtain new types of solutions to the Eq. (2). For this purpose, the Jacobi elliptic functions expansion method is utilized. This method introduces different forms of solutions at once. In the application, it is seen that the mentioned method is indeed fertile compared to other methods in the literature. 


\section{Preliminaries}

There are some significant definitions for the fractional derivative, such as the Riemann-Liouville fractional derivative [25], Caputo's fractional derivative [26], and conformable fractional derivative [27].

Now, we give the basic definition of the conformable fractional derivative and some important features.

Let $u(x):[0, \infty] \rightarrow R$ be a function. Then the conformable fractional derivative of the function $u(x)$ is given by

$$
D_{x}^{\gamma} u(x)=\lim _{h \rightarrow 0} \frac{u\left(x+h x^{1-\gamma}\right)-u(x)}{h}, \quad 0<\gamma \leq 1, \quad x>0 .
$$

The function $u(x)$ is called $\gamma$-differentiable or has $\gamma$ order fractional derivative if the limit (3) exists.

Theorem 1 Suppose that $u(x)$ and $v(x)$ are $\gamma$-differentiable, $0<\gamma \leq 1$, and $a$, c are constants. Then the following equalities are satisfied:

$$
\begin{aligned}
& -\quad D_{x}^{\gamma}(c)=0, \\
& \text { - } D_{x}^{\gamma}(c u(x))=c D_{x}^{\gamma}(u(x)), \\
& \text { - } D_{x}^{\gamma}\left(x^{n}\right)=n x^{n-\gamma}, \\
& \text { - } \quad D_{x}^{\gamma}(a u(x)+b v(x))=a D_{x}^{\gamma}(u(x))+c D_{x}^{\gamma}(v(x)), \\
& \text { - } \quad D_{x}^{\gamma}(u(x) v(x))=v(x) D_{x}^{\gamma}(u(x))+u(x) D_{x}^{\gamma}(v(x)), \\
& \text { - } D_{x}^{\gamma}\left(\frac{u(x)}{v(x)}\right)=\frac{v(x) D_{x}^{\gamma}(u(x))-u(x) D_{x}^{\gamma}(v(x))}{v^{2}(x)}, \quad v(x) \neq 0, \\
& \text { - } \quad D_{x}^{\gamma}(u(x))=x^{1-\gamma} \frac{d u}{d x},
\end{aligned}
$$

where $u(x)$ is a first order differentiable function.

\section{The Jacobi elliptic functions expansion method}

To understand how this method works, we give a summary of the method. In this methodology, we look for the solutions of the following nonlinear fractional order partial differential equation:

$$
Q\left(u, D_{t}^{\gamma} u, D_{x}^{\beta} u, D_{t}^{\gamma} D_{x}^{\beta} u, D_{t t}^{2 \gamma} u, D_{x x}^{2 \beta} u, \ldots\right)=0,
$$

where $\gamma$ and $\beta$ are the fractional orders. 
For the Schrödinger equation, the wave transformation is considered as follows:

$$
u(x, t)=v(\xi) e^{i\left(w \frac{x^{2} \beta}{2 \beta}-\lambda \frac{t^{\gamma}}{\gamma}\right)}, \quad \xi=\frac{x^{2 \beta}}{2 \beta}-2 w \frac{t^{\gamma}}{\gamma}
$$

where $\lambda$ is a constant.

Then, putting the equation (5) into the equation (4) converts it to the following ODE with respect to the variable $\xi$,

$$
P\left(v, v^{\prime}, v^{\prime \prime}, \ldots\right)=0
$$

where $P$ is a polynomial of the functions $v, v^{\prime}, v^{\prime \prime}, \ldots$.

For different kinds of solutions to (6), we investigate the solutions in the following forms:

$$
\begin{aligned}
& v(\xi)=a_{0}+\sum_{j=1}^{n} a_{j} s n^{j}(k \xi \mid m)+\sum_{j=1}^{n} b_{j} n s^{j}(k \xi \mid m), \\
& v(\xi)=a_{0}+\sum_{j=1}^{n} a_{j} c n^{j}(k \xi \mid m)+\sum_{j=1}^{n} b_{j} n c^{j}(k \xi \mid m),
\end{aligned}
$$

and

$$
v(\xi)=a_{0}+\sum_{j=1}^{n} a_{j} d n^{j}(k \xi \mid m)+\sum_{j=1}^{n} b_{j} n d^{j}(k \xi \mid m)
$$

where $n>0$ is a desired positive parameter and named as the balancing constant.

\section{Solutions of fractional Schrödinger equation}

We consider the equation in the following form:

$$
i D_{t}^{\gamma} u+D_{x}^{2 \beta} u+\mu|u|^{2} u=0, \quad 0<\gamma, \beta \leq 1, \quad t>0,
$$

where $u(x, t)$ is a complex-valued function and $\mu \neq 0$ is a real constant. The wave transformation (5) turns the equation (10) to the following form:

$$
v^{\prime \prime}+\left(\lambda-w^{2}\right) v+\mu v^{3}=0 .
$$

We use the expansions (7)-(9) in order to obtain the solutions of the equation (11). We get the balancing constant as $n=1$ from the equation (11).

\subsection{The sn-ns method}

In this part, we utilize the Jacobi elliptic sine function expansion to acquire the solution of the equation (11) as follows: 


$$
v(\xi)=a_{0}+a_{1} \operatorname{sn}(k \xi \mid m)+b_{1} n s(k \xi \mid m),
$$

where $\operatorname{sn}(k \xi \mid m)$ represents the Jacobi elliptic sine function, and $n s(k \xi \mid m)=s n^{-1}(k \xi \mid m)$ is the inverse function.

Putting the equation (12) into the equation (11) yields

$$
\begin{aligned}
& 3 a_{0} a_{1}^{2} \mu=0, \\
& a_{1}^{3} \mu+a_{1} k^{2} m^{2}+a_{1} k^{2} m=0, \\
& a_{1}\left(\lambda-w^{2}\right)+3 a_{1}^{2} b_{1} \mu+3 a_{0}^{2} a_{1} \mu-a_{1} k^{2} m^{2}-a_{1} k^{2}=0, \\
& b_{1}\left(\lambda-w^{2}\right)+3 a_{0}^{2} b_{1} \mu+3 a_{1} b_{1}^{2} \mu-b_{1} k^{2} m^{2}-b_{1} k^{2}=0, \\
& a_{0}\left(\lambda-w^{2}\right)+a_{0}^{3} \mu+6 a_{0} a_{1} b_{1} \mu=0, \\
& 3 a_{0} b_{1}^{2} \mu=0, \\
& b_{1}^{3} \mu+2 k^{2} b_{1}=0 .
\end{aligned}
$$

Solving these system gives the desired unkowns as follows:

$$
\begin{aligned}
& a_{0}=0, \quad a_{1}=\mp \frac{\sqrt{-\left(\lambda-w^{2}\right)\left(m^{2}+m\right)}}{\sqrt{\mu\left(m^{2}+1\right)}}, \quad b_{1}=0, \quad k=\mp \frac{\sqrt{\left(\lambda-w^{2}\right)}}{\sqrt{m^{2}+1}}, \\
& a_{0}=0, \quad a_{1}=0, \quad b_{1}=\mp \frac{\sqrt{2\left(\lambda-w^{2}\right)}}{\sqrt{-\mu\left(m^{2}+1\right)}}, \quad k=\mp \frac{\sqrt{\left(\lambda-w^{2}\right)}}{\sqrt{m^{2}+1}} .
\end{aligned}
$$

Putting these into the expansion (12) provides

$$
\begin{aligned}
& v_{1}(\xi)=\mp \frac{\sqrt{-\left(\lambda-w^{2}\right)\left(m^{2}+m\right)}}{\sqrt{\mu\left(m^{2}+1\right)}} s n\left(\mp \frac{\sqrt{\left(\lambda-w^{2}\right)}}{\sqrt{m^{2}+1}} \xi \mid m\right), \\
& v_{2}(\xi)=\mp \frac{\sqrt{2\left(\lambda-w^{2}\right)}}{\sqrt{-\mu\left(m^{2}+1\right)}} n s\left(\mp \frac{\sqrt{\left(\lambda-w^{2}\right)}}{\sqrt{m^{2}+1}} \xi \mid m\right) .
\end{aligned}
$$

Then we have the solutions for the Schrödinger equation given in (10) as

$$
u_{1,2}(\xi)=\mp \frac{\sqrt{-\left(\lambda-w^{2}\right)\left(m^{2}+m\right)}}{\sqrt{\mu\left(m^{2}+1\right)}} s n\left(\mp \frac{\sqrt{\left(\lambda-w^{2}\right)}}{\sqrt{m^{2}+1}} \xi \mid m\right) e^{i\left(w^{\frac{x^{2} \beta}{2 \beta}}-\lambda \frac{t^{\gamma}}{\gamma}\right)},
$$




$$
u_{3,4}(\xi)=\mp \frac{\sqrt{2\left(\lambda-w^{2}\right)}}{\sqrt{-\mu\left(m^{2}+1\right)}} n s\left(\mp \frac{\sqrt{\left(\lambda-w^{2}\right)}}{\sqrt{m^{2}+1}} \xi \mid m\right) e^{i\left(w^{\frac{x^{2} \beta}{2 \beta}}-\lambda \frac{t^{\gamma}}{\gamma}\right)},
$$

where $\xi=\frac{x^{2 \beta}}{2 \beta}-2 w \frac{t^{\gamma}}{\gamma}$.

In addition to these ones, we reach the following solutions for $m \rightarrow 1$ and $m \rightarrow 0$, respectively.

$$
\begin{aligned}
& u_{5,6}(\xi)=\mp \sqrt{\frac{w^{2}-\lambda}{\mu}} \tanh \left(\frac{\sqrt{\lambda-w^{2}}}{\sqrt{2}}\left(\frac{x^{2 \beta}}{2 \beta}-2 w \frac{t^{\gamma}}{\gamma}\right)\right) e^{i\left(w x^{2 \beta} \frac{x^{2}}{2 \beta}-\lambda \frac{t \gamma}{\gamma}\right)}, \\
& u_{7,8}(\xi)=\mp \sqrt{\frac{w^{2}-\lambda}{\mu}} \operatorname{coth}\left(\frac{\sqrt{\lambda-w^{2}}}{\sqrt{2}}\left(\frac{x^{2 \beta}}{2 \beta}-2 w \frac{t \gamma}{\gamma}\right)\right) e^{i\left(w \frac{x^{2} \beta}{2 \beta}-\lambda \frac{t^{\gamma}}{\gamma}\right)},
\end{aligned}
$$

and

$$
u_{9,10}(\xi)=\mp \sqrt{\frac{2\left(w^{2}-\lambda\right)}{\mu}} \csc \left(\sqrt{\lambda-w^{2}}\left(\frac{x^{2 \beta}}{2 \beta}-2 w \frac{t^{\gamma}}{\gamma}\right)\right) e^{i\left(w \frac{x^{2 \beta}}{2 \beta}-\lambda \frac{t \gamma}{\gamma}\right)} .
$$

\subsection{The cn-nc method}

In this section, we use the Jacobi elliptic cosine function expansion to obtain the solutions in the following form:

$$
v(\xi)=a_{0}+a_{1} c n(k \xi \mid m)+b_{1} n c(k \xi \mid m),
$$

where $c n(k \xi \mid m)$ stands for the Jacobi elliptic cosine function, and $n c(k \xi \mid m)=c n^{-1}(k \xi \mid m)$ is the inverse function.

Following the same process above, we obtain

$$
\begin{aligned}
& u_{11,12}(\xi)=\mp \frac{\sqrt{-m\left(\lambda-w^{2}\right)}}{\sqrt{\mu}} c n\left(\mp \frac{\sqrt{\left(\lambda-w^{2}\right)}}{\sqrt{m+1}} \xi \mid m\right) e^{i\left(w \frac{x^{2 \beta}}{2 \beta}-\lambda \frac{\gamma}{\gamma}\right)}, \\
& u_{13,14}(\xi)=\mp \frac{\sqrt{2\left(\lambda-w^{2}\right)}}{\sqrt{-\mu\left(m^{2}+1\right)}} n c\left(\mp \frac{\sqrt{\left(\lambda-w^{2}\right)}}{\sqrt{m^{2}+1}} \xi \mid m\right) e^{i\left(w \frac{x^{2 \beta}}{2 \beta}-\lambda \frac{t^{\gamma} \gamma}{\gamma}\right)},
\end{aligned}
$$

where $\xi=\frac{x^{2 \beta}}{2 \beta}-2 w \frac{t^{\gamma}}{\gamma}$. 
For $m \rightarrow 1$, we have

$$
\begin{aligned}
& u_{15,16}(\xi)=\mp \sqrt{\frac{w^{2}-\lambda}{\mu}} \operatorname{sech}\left(\frac{\sqrt{\lambda-w^{2}}}{\sqrt{2}}\left(\frac{x^{2 \beta}}{2 \beta}-2 w \frac{t \gamma}{\gamma}\right)\right) e^{i\left(w \frac{x^{2} \beta}{2 \beta}-\lambda \frac{t^{\gamma}}{\gamma}\right)}, \\
& u_{17,18}(\xi)=\mp \sqrt{\frac{w^{2}-\lambda}{\mu}} \cosh \left(\frac{\sqrt{\lambda-w^{2}}}{\sqrt{2}}\left(\frac{x^{2 \beta}}{2 \beta}-2 w \frac{t^{\gamma}}{\gamma}\right)\right) e^{i\left(w \frac{x^{2 \beta}}{2 \beta}-\lambda \frac{t^{\gamma}}{\gamma}\right)} .
\end{aligned}
$$

For $m \rightarrow 0$, we get

$$
u_{19,20}(\xi)=\mp \sqrt{\frac{2\left(w^{2}-\lambda\right)}{\mu}} \sec \left(\sqrt{\lambda-w^{2}}\left(\frac{x^{2 \beta}}{2 \beta}-2 w \frac{t^{\gamma}}{\gamma}\right)\right) e^{i\left(w \frac{x^{2 \beta}}{2 \beta}-\lambda \frac{\gamma}{\gamma}\right)} .
$$

\subsection{The dn-nd method}

In this chapter, we look for the solutions as in the following form:

$$
v(\xi)=a_{0}+a_{1} d n(k \xi \mid m)+b_{1} n d(k \xi \mid m),
$$

where $d n(k \xi \mid m)$ is named as the delta amplitude.

Using the same procedure mentioned above provides us with the following solutions:

$$
\begin{gathered}
u_{21,22}(\xi)=\mp \frac{\sqrt{-2\left(\lambda-w^{2}\right)}}{\sqrt{\mu\left(m^{2}-2\right)}} d n\left(\mp \frac{\sqrt{m\left(\lambda-w^{2}\right)}}{\sqrt{m^{2}-2}} \xi \mid m\right) e^{i\left(w \frac{x^{2 \beta}}{2 \beta}-\lambda \frac{\gamma}{\gamma}\right)} \\
u_{23,24}(\xi)=\mp \frac{\sqrt{\left(m^{2}+m-2\right)\left(\lambda-w^{2}\right)}}{\sqrt{\mu(2-m)}} n d\left(\mp \frac{\sqrt{m\left(\lambda-w^{2}\right)}}{\sqrt{m-2}} \xi \mid m\right) e^{i\left(w \frac{x^{2 \beta}}{2 \beta}-\lambda \frac{\gamma}{\gamma}\right)},
\end{gathered}
$$

where $\xi=\frac{x^{2 \beta}}{2 \beta}-2 w \frac{t^{\gamma}}{\gamma}$.

Letting $m \rightarrow 1$ and $m \rightarrow 0$ gives the following solutions, respectively;

$$
\begin{gathered}
u_{25,26}(\xi)=\mp \sqrt{\frac{2\left(w^{2}-\lambda\right)}{\mu}} \operatorname{sech}\left(\sqrt{\lambda-w^{2}}\left(\frac{x^{2 \beta}}{2 \beta}-2 w \frac{t^{\gamma}}{\gamma}\right)\right) e^{i\left(w \frac{x^{2 \beta}}{2 \beta}-\lambda \frac{t^{\gamma}}{\gamma}\right)}, \\
u_{27,28}(\xi)=\mp \sqrt{\frac{w^{2}-\lambda}{\mu}} e^{i\left(w \frac{x^{2 \beta}}{2 \beta}-\lambda \frac{\gamma \gamma}{\gamma}\right)} .
\end{gathered}
$$




\section{Graphical representaion}

Now, we illustrate the solutions depending on the order of fractional derivative, namely $\gamma$ and $\beta$ in 2D by setting $x=10$ (Figs. 1-16).
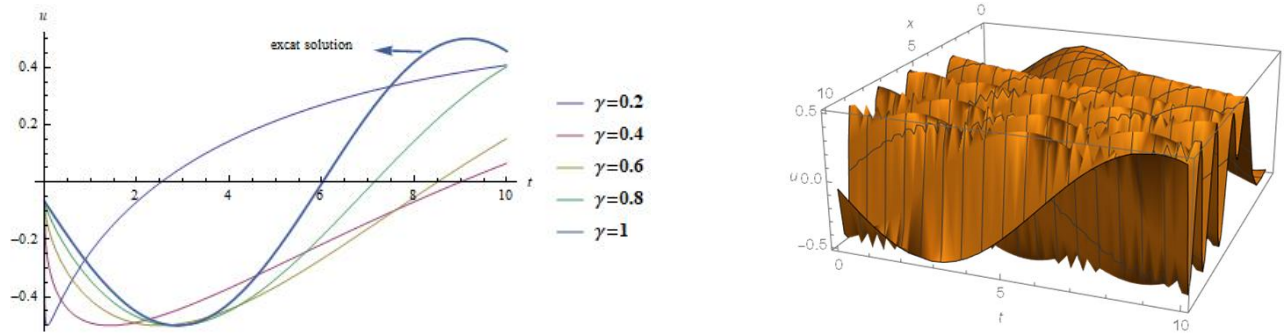

Fig. 1. Real part of Eq. (15), $x=10, \lambda=0.5, w=0.5, \mu=1, \beta=1$
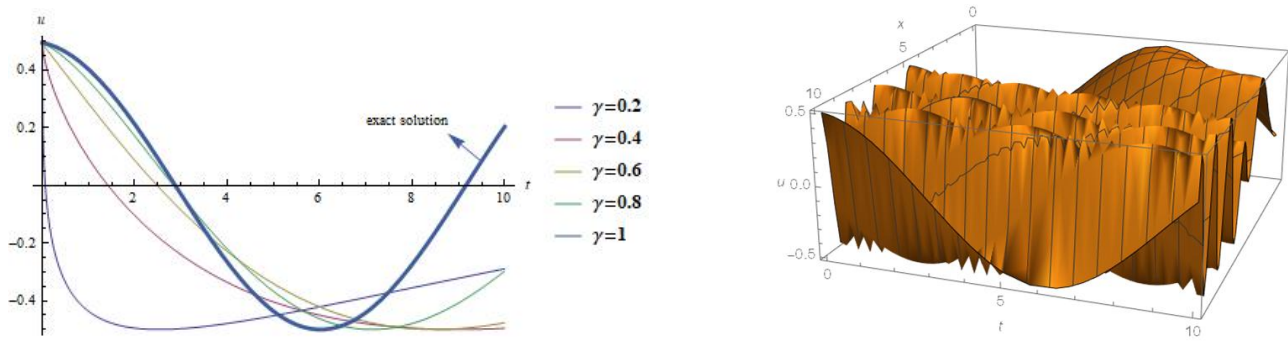

Fig. 2. Imaginary part of Eq. (15), $x=10, \lambda=0.5, w=0.5, \mu=1, \beta=1$
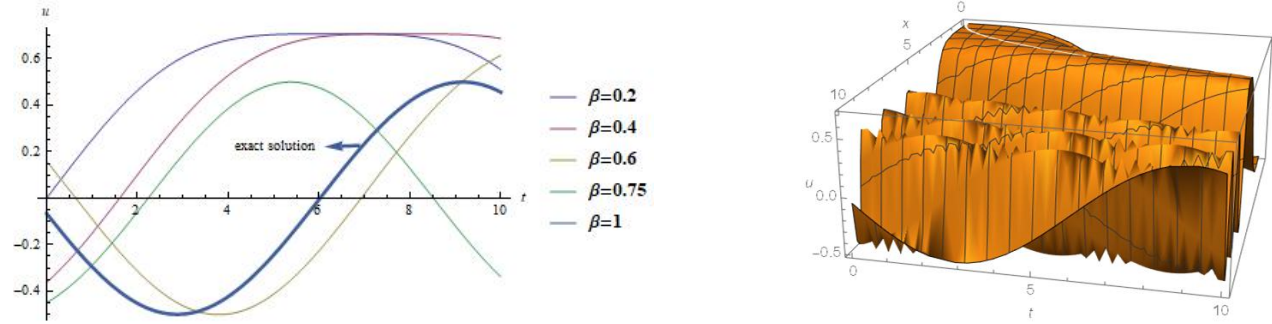

Fig. 3. Real part of Eq. (16), $x=10, \lambda=0.5, w=0.5, \mu=1, \gamma=1$ 

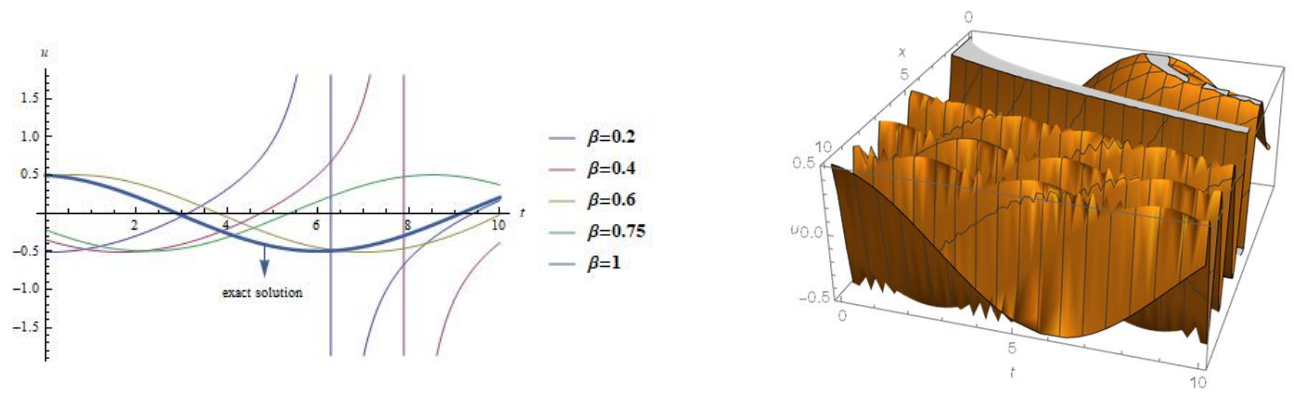

Fig. 4. Imaginary part of Eq. (16), $x=10, \lambda=0.5, w=0.5, \mu=1, \gamma=1$
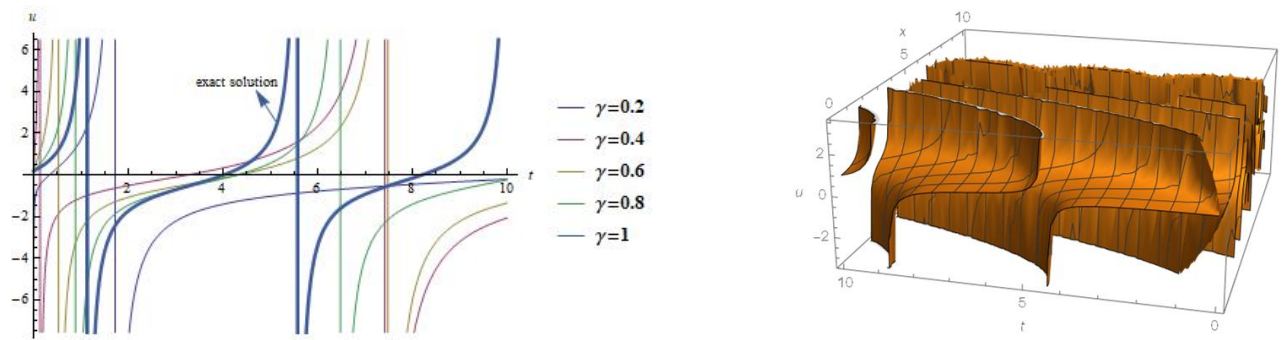

Fig. 5. Real part of Eq. (17), $x=10, \lambda=0.75, w=0.5, \mu=1, \beta=1$
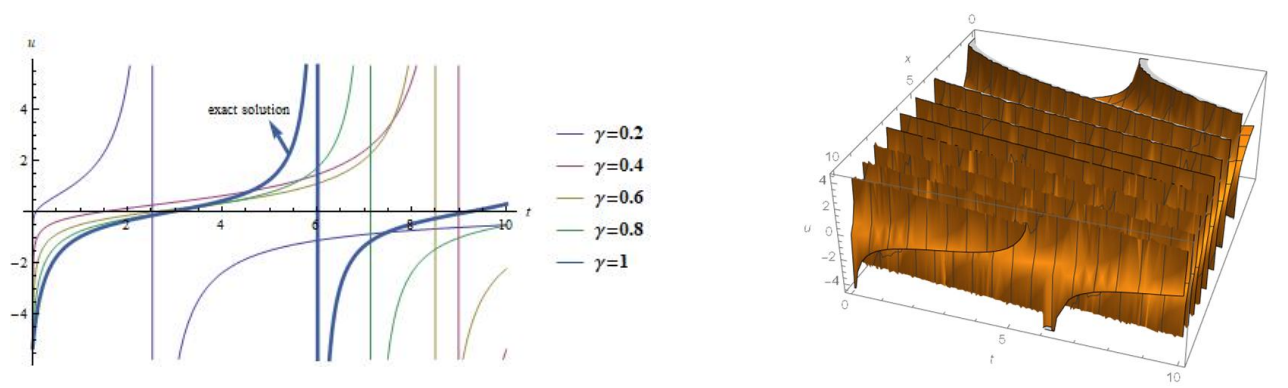

Fig. 6. Imaginary part of Eq. (17), $x=10, \lambda=0.5, w=0.5, \mu=1, \beta=1$
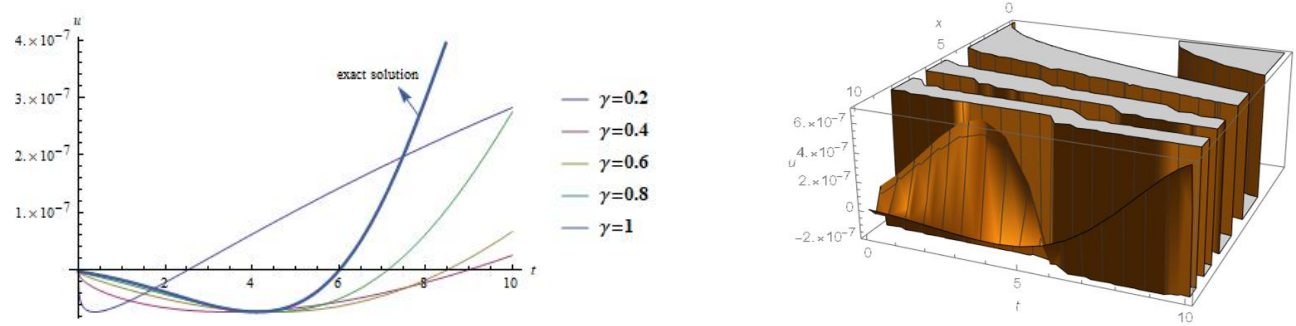

Fig. 7. Real part of Eq. (21), $x=10, \lambda=0.5, w=0.5, \mu=1, \beta=1$

The illustrations, therefore, show the changes of the solutions over time. Moreover, we represent the exact solutions in 3D.

In Figures 3 and 4 , we take $\gamma=1$ and see how the solutions act with respect to the values of $\beta$. As shown in these figures, we reach the exact solution when $\beta$ goes to 1 . 

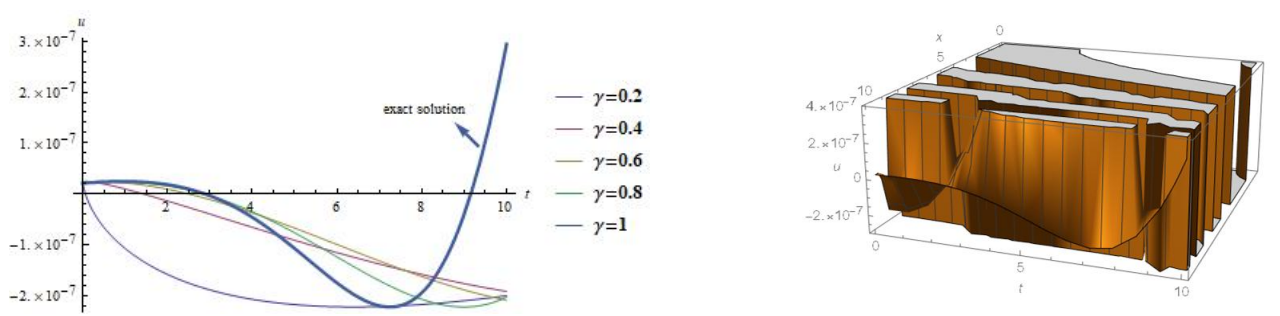

Fig. 8. Imaginary part of Eq. (21), $x=10, \lambda=0.5, w=0.5, \mu=1, \beta=1$
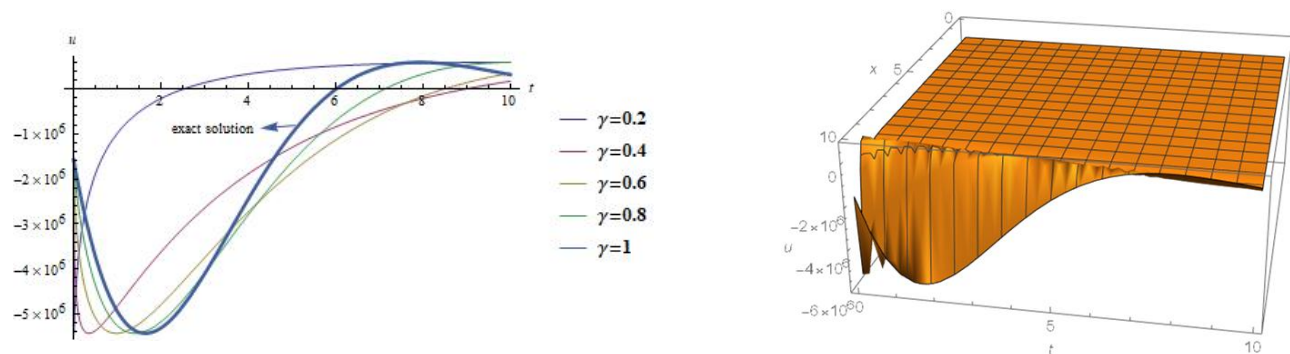

Fig. 9. Real part of Eq. (22), $x=10, \lambda=0.5, w=0.5, \mu=1, \beta=1$
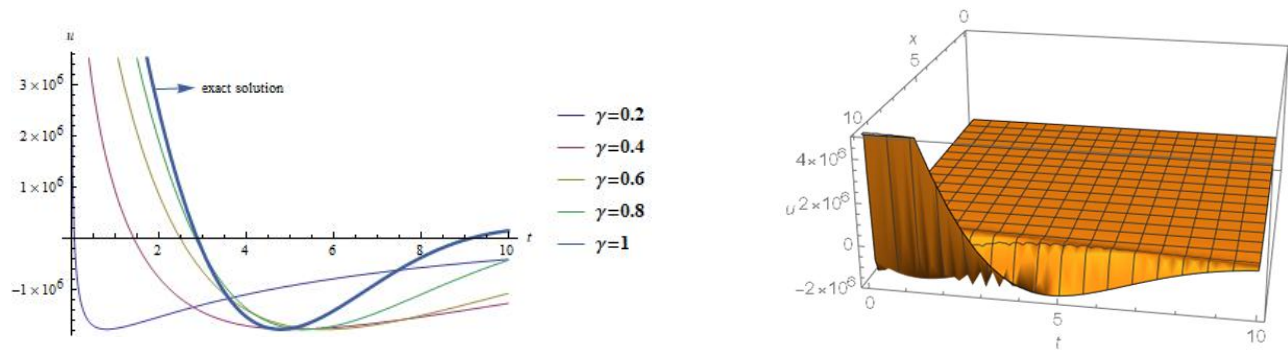

Fig. 10. Imaginary part of Eq. (22), $x=10, \lambda=0.5, w=0.5, \mu=1, \beta=1$
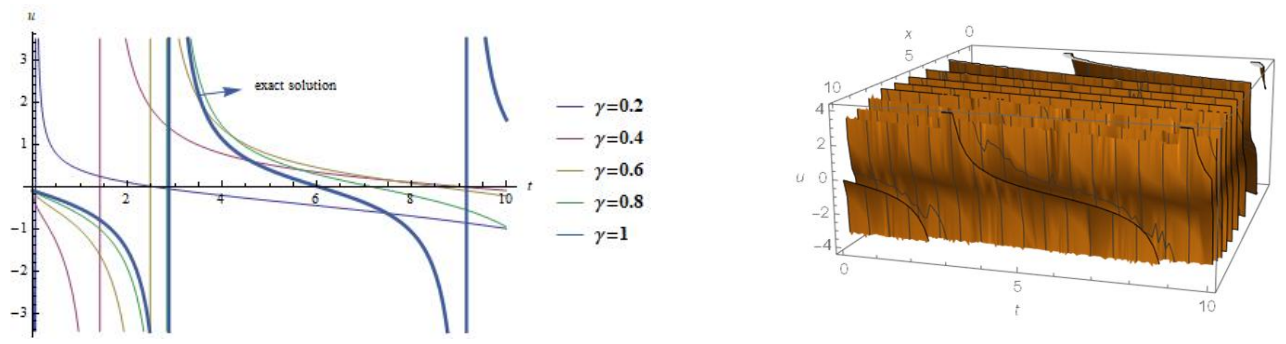

Fig. 11. Real part of Eq. (23), $x=10, \lambda=0.5, w=0.5, \mu=1, \beta=1$

For the others, we set $\beta=1$ and plot the graphics of the solutions depending on the $\gamma$. Similarly, we gain the exact solutions when $\gamma$ tends to 1 . 

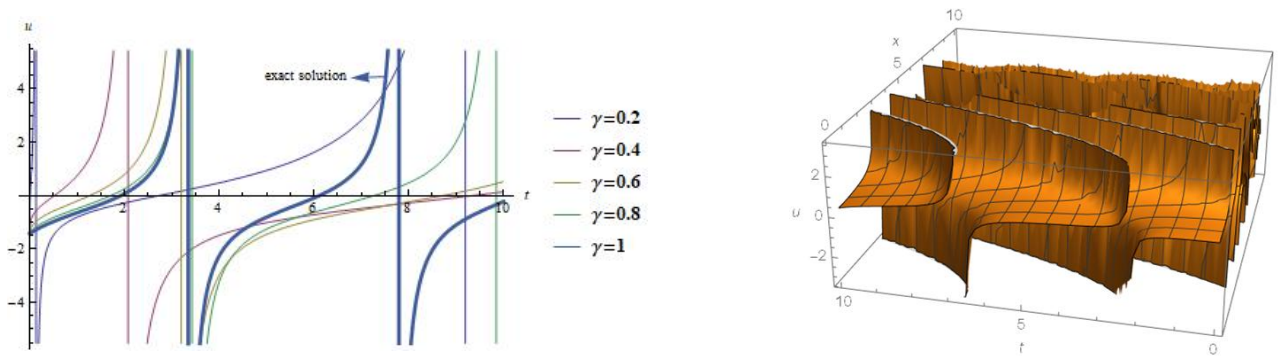

Fig. 12. Imaginary part of Eq. (23), $x=10, \lambda=0.75, w=0.5, \mu=1, \beta=1$
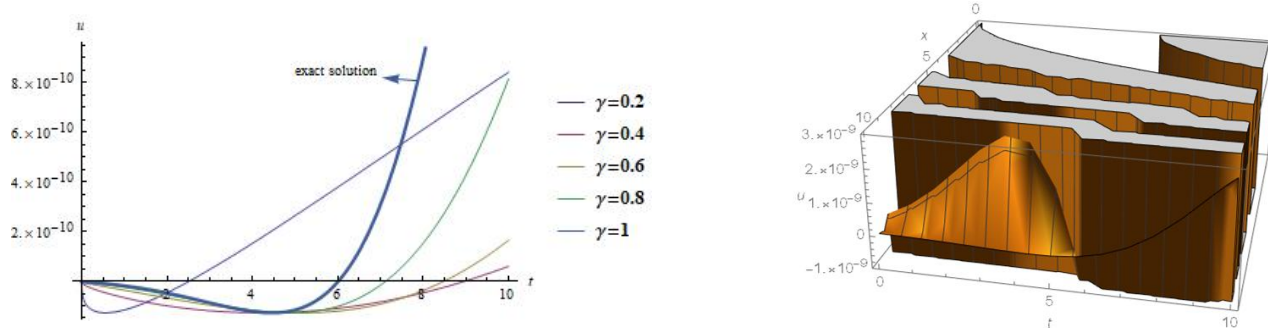

Fig. 13. Real part of Eq. (27), $x=10, \lambda=0.5, w=0.5, \mu=1, \beta=1$
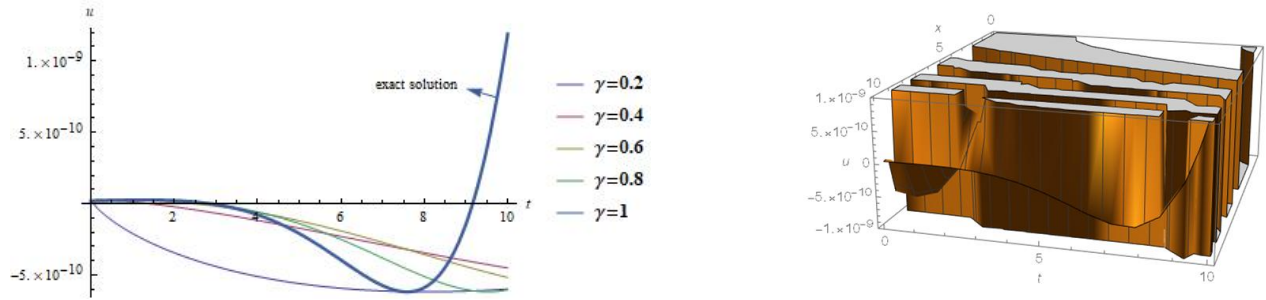

Fig. 14. Imaginary part of Eq. (27), $x=10, \lambda=0.5, w=0.5, \mu=1, \beta=1$
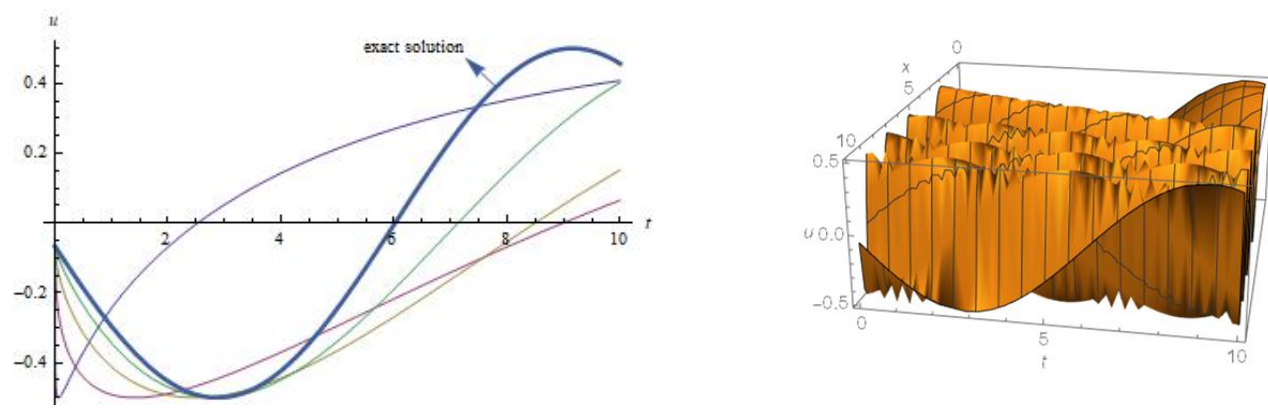

Fig. 15. Real part of Eq. (28), $x=10, \lambda=0.5, w=0.5, \mu=1, \beta=1$

\section{Conclusion}

The main purpose of this present paper is to put forth new types of solutions to cubic nonlinear time and space fractional Schrödinger equation in a conformable sense. Using the wave transformation given in (5), the Schrödinger equation (10) is 

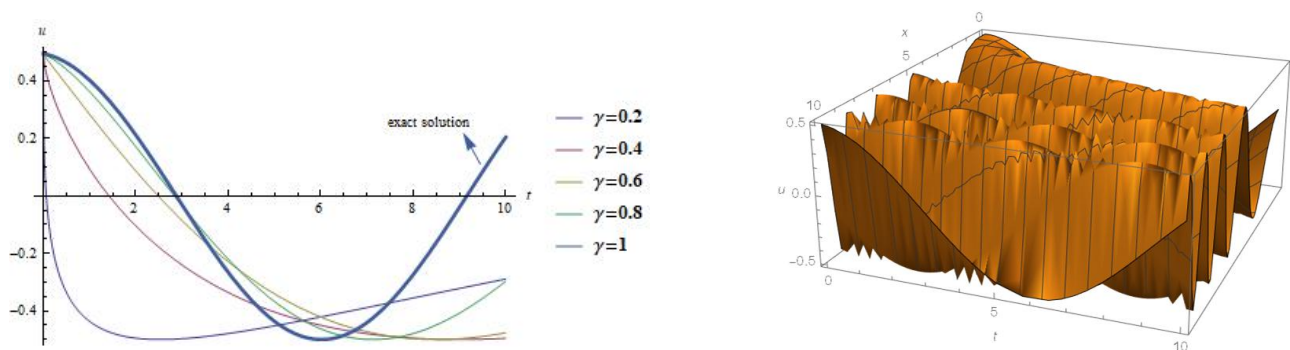

Fig. 16. Imaginary part of Eq. (28), $x=10, \lambda=0.5, w=0.5, \mu=1, \beta=1$

converted into ordinary differential equation w.r.t $\xi$. One of the powerful methods for solving ODEs, the Jacobi elliptic functions expansion method, is implemented to the ODE (11). This method introduces new travelling wave solutions to the mentioned equation including Jacobi elliptic functions solutions, hyperbolic solutions, and trigonometric solutions. In addition to these forms of solutions, the kink solution, soliton solution, periodic solutions, and singular solutions are obtained. As far as we know, the presented solutions to cubic nonlinear time and space fractional Schrödinger equation in a conformable sense have not been noticed in the open literature. Finally, the findings in this paper demonstrate how this method deals with the time-space fractional PDEs as well as ordinary ones.

\section{References}

[1] Weitzner, H., \& Zaslavskyn, G.M. (2003). Some applications of fractional equations. Commun. Nonlinear Sci. Numer. Simulat., 8, 273-281.

[2] Kilbas, A., Srivastava, H., \& Trujillo, J. (2006). Theory and Applications of Fractional Differential Equations. Elsevier.

[3] Baleanu, D., Golmankhaneh, A.K., \& Baleanu, M.C. (2009). Fractional electromagnetic equations using fractional forms. International Journal of Theoretical Physics, 48, 3114-3123.

[4] Wazwaz, A. (2007). The extended tanh method for new solitons solutions for many forms of the fifth-order KdV equations. Applied Mathematics and Computation, 184, 1002-1014.

[5] Gözükızıl, O.F., \& Akcagil, S. (2013). The tanh-coth method for some nonlinear pseudoparabolic equations with exact solutions. Advances in Difference Equations, DOI: 10.1186/1687-18472013-143.

[6] He, J., \& Zhang, L. (2008). Generalized solitary solution and compacton-like solution of the Jaulent-Miodek equations using the Exp-function method. Physical Letters A, 371, 1044-1047.

[7] Wang, M., Li, X., \& Zahng, J. (2008). The (G'/G)-expansion method and travelling wave solutions of nonlinear evolution equations in mathematical physics. Physical Letters A, 372, 417-423.

[8] Akcagil, S., Aydemir, T., \& Gözükızıl, O.F. (2015). Comparison between the new (G'/G) expansion method and the extended homogeneous balance method. New Trends in Mathematical Sciences, 3, 223-236.

[9] Gündoğdu, H., \& Gözükızıl, O.F. (2017). Solving nonlinear partial differential equations by using Adomian decomposition method, modified decomposition method and Laplace decomposition method. MANAS Journal of Engineering, 5, 1-13. 
[10] Gündoğdu, H., \& Gözükızıl, O.F. (2017). Obtaining the solution of Benney-Luke Equation by Laplace and adomian decomposition methods. Sakarya University Journal of Science, 21, $1524-1528$

[11] Alvaro, H.S. (2012). Solving nonlinear partial differential equations by the sn-ns method. Abstr. Appl. Analysis, 25, 1-25.

[12] Gündoğdu, H., \& Gözükızıl, O.F. (2021). On the new type of solutions to Benney-Luke equation. BSPM - Sociedade Paranaense de Matemática, DOI: 10.5269/bspm.41244.

[13] Gündoğdu, H., \& Gözükızıl, O.F., (2018). On different kinds of solutions to simplified modified form of the Camassa-Holm equation. Journal of Applied Mathematics and Computational Mechanics, 17, 1-10.

[14] Laskin, N. (2002). Fractional Schrödinger equation. Phys. Rev., 66, 056108.

[15] Biswas, A. (2012). Soliton solutions of the perturbed resonant nonlinear dispersive Schrödinger's equation with full nonlinearity by a semi-inverse variational principle. Quantum Phys. Lett., 1, 79-84.

[16] Eslami, M., Mirzazadeh, M., \& Biswas, A. (2013). Soliton solutions of the resonant nonlinear Schrödinger's equation in optical fibers with time-dependent coefficients by simplest equation approach. J. Mod. Opt., 60, 1627-1636.

[17] Mirzazadeh, M., Eslami, M., Milovic, D., \& Biswas, A. (2014). Topological solitons of resonant nonlinear Schrödinger's equation with dual-power law nonlinearity using (G'/G)-expansion technique. Optik, 125, 5480-5489.

[18] Inc, M., \& Ates, E. (2017). Bright, dark and singular optical solitons in a power-law media with fourth-order dispersion. Opt. Quantum Electron., DOI: 10.1007/s11082-017-1150-0.

[19] Akbulut, A., \& Kaplan, M. (2018). Auxiliary equation method for time-fractional differential equations with conformable derivative. Comput. Math Appl., 75, 876-882.

[20] Owyed, S., Abdou, M.A., Abdel-Aty, A., \& Dutta, H. (2019). Optical solitons solutions for perturbed time fractional nonlinear Schrodinger equation via two strategic algorithms. AIMS Mathematics, 5, 2057-2070.

[21] Neirameh, A., Eslami, M., \& Mehdipoor M. (2021). New Types of Soliton Solutions for Spacetime Fractional Cubic Nonlinear Schrodinger Equation. Bol. Soc. Paran. Mat., 39, 121-131.

[22] Laskin, N. (2000). Fractional quantum mechanics. Phys. Rev., 62, 3135-3145.

[23] Laskin, N. (2000). Fractional quantum mechanics and Levy path integrals. Phys. Lett. A., 268-298.

[24] Laskin, N. (2000). Fractals and quantum mechanics. Chaos, 10, 780.

[25] Kilbas, A.A., Srivastava H.M., \& Trujillo J.J. (2006). Theory and Applications of Fractional Differential Equations. Amsterdam: Elsevier.

[26] Debnath L. (2003). Recent applications of fractional calculus to science and engineering. Int. J. Math. Math. Sci., 54, 3413-3442.

[27] Khalil, R., Al Horani, M., Yousef, A., \& Sababheh, M. (2014). A new definition of fractional derivative. Journal of Computational and Applied Mathematics, 264, 65-70. 